H. Ito

Nagoya Math. J.

Vol. 118 (1990), 35-43

\title{
DEDEKIND SUMS AND QUADRATIC RESIDUE SYMBOLS
}

\author{
HIROSHI ITO
}

1. In this paper we first prove a simple relation between sums of a certain type and quadratic residue symbols. Then we apply this to Dedekind sums introduced by Sczech [5]. In particular one of his conjectures in [6] will be proved.

2. We will consider congruence relations such as

$$
a \equiv b \quad\left(\bmod 2^{n}\right), \quad n \geq 1,
$$

where $a$ and $b$ are algebraic (not necessarily integral) numbers. We take this to mean that $a-b=2^{n} c$ with a 2-integral algebraic number $c$. Let $K$ be an algebraic number field of finite degree, $\mathfrak{o}$ its ring of integers, and $c$ an integral ideal of $K$ prime to 2 . Denote by $N \mathfrak{c}$ the absolute norm of $c$. Let $f$ be a map from $\mathfrak{o} / \mathfrak{C}-\{0\}$ to an algebraic number field containing $K$.

Proposition 1. If $f$ satisfies the conditions

$$
\begin{aligned}
& f(-k)=-f(k), \\
& f(k) \equiv 1 \quad(\bmod 2),
\end{aligned}
$$

then, for every $a \in \mathfrak{D}$ prime to $\mathfrak{c}$,

$$
\sum_{\substack{k \in \mathcal{O} / \mathfrak{c} \\ k \neq 0}} f(a k) f(k) \equiv N c+1-2\left(\frac{a}{\mathfrak{c}}\right) \quad(\bmod 8) .
$$

Here $(a / c)$ is the quadratic residue symbol of $K$.

Proof. Let $R$ be a subset of $\mathfrak{o} / \mathfrak{c}$ such that $R \cap(-R)=\varnothing$ and $\mathfrak{o} / \mathfrak{c}=$ $R \cup(-R) \cup\{0\}$. By (1),

$$
\begin{aligned}
& (f(-a k)-1)(f(-k)+1) \\
= & (f(a k)-1)(f(k)+1)+2(f(k)-f(a k)) .
\end{aligned}
$$

Therefore, from (2),

Received March 22, 1988.

The author was supported by NSF Grant DMS-8601978. 


$$
\begin{aligned}
& \sum_{\substack{k \in \mathbb{1}(\mathrm{C} \\
k \neq 0}}(f(a k)-1)(f(k)+1) \\
& \quad=2 \sum_{k \in R}\{(f(a k)-1)(f(k)+1)+f(k)-f(a k)\} \\
& \quad \equiv 2\left\{\sum_{k \in R} f(k)-\sum_{k \in a R} f(k)\right\} \quad(\bmod 8) .
\end{aligned}
$$

Put

$$
R_{n}=R \cap(-1)^{n} a R, \quad n=0,1 .
$$

Then $R=R_{0} \cup R_{1}$ and $a R=R_{0} \cup\left(-R_{1}\right)$, the unions being disjoint. Therefore, by (1) and (2),

$$
\begin{aligned}
& 2\left\{\sum_{k \in R} f(k)-\sum_{k \in a R} f(k)\right\} \\
& \quad=4 \sum_{k \in R_{1}} f(k) \\
& \quad \equiv 4 \cdot \# R_{1} \quad(\bmod 8) .
\end{aligned}
$$

A generalization of Gauss' lemma (cf. Reichardt [4]) says

$$
\# R_{1} \equiv \frac{1}{2}\left(1-\left(\frac{a}{c}\right)\right) \quad(\bmod 2) .
$$

Because (3) is equal to

$$
\sum_{\substack{k \in \in=1 \mathrm{c} \\ k \neq 0}} f(a k) f(k)-N \mathrm{c}+1
$$

we have proved the proposition.

Note that, for every odd integer $n$,

$$
n-1 \equiv 2\left(1-(-1)^{\left(n^{2}-1\right) / 8}\right)-1+(-1)^{(n-1) / 2} \quad(\bmod 8) .
$$

Then the congruence of Proposition 1 can be written as

$$
\sum_{\substack{k \in=0 / c \\ k \neq 0}} f(a k) f(k) \equiv 2\left(1-\left(\frac{2 a}{c}\right)\right)-1+\left(\frac{-1}{c}\right) \quad(\bmod 8) .
$$

We also remark that the condition (1) can be replaced by

$$
f(-k) \equiv-f(k) \quad(\bmod 4) .
$$

3. Example. We apply Proposition 1 with $K=\boldsymbol{Q}$ and $c=c \boldsymbol{Z}, c$ being an odd integer. Define

$$
f_{1}(k)=2\left(\left(\frac{k}{c}\right)\right), \quad f_{2}(k)=i^{-1} \cot \left(\pi \frac{k}{c}\right)
$$


for every integer $k$ not divisible by $c$. Here

$$
((x))=x-[x]-\frac{1}{2}, \quad x \in R-Z
$$

with $[x]$ the greatest integer not exceeding $x$. It is easy to see that both of $f_{1}$ and $f_{2}$, viewed as functions on $Z / c Z-\{0\}$, satisfy the conditions for $f$ in Proposition 1. Therefore,

$$
\begin{aligned}
& 4 \sum_{\substack{k \in Z \mid c Z \\
k \neq 0}}\left(\left(\frac{a k}{c}\right)\right)\left(\left(\frac{k}{c}\right)\right) \\
& \equiv-\sum_{\substack{k \in Z / c Z \\
k \neq 0}} \cot \left(\pi \frac{a k}{c}\right) \cot \left(\pi \frac{k}{c}\right) \\
& \equiv|c|+1-2\left(\frac{a}{c}\right) \quad(\bmod 8) .
\end{aligned}
$$

These congruences are well-known (cf. Rademacher and Grosswald [3]).

4. In the following $K$ denotes an imaginary quadratic field with discriminant $D$ and o the ring of integers of $K$. We fix an embedding of $K$ into $C$. Here we recall some known facts contained in [5]. Let $L$ be a lattice in $C$ such that $\mathfrak{o}=\{m \in C ; m L \subset L\}$ and let, for $z \in C$ and $n \in Z$, $n \geq 0$,

$$
E_{n}(z)=E_{n}(z, L)=\left.\sum_{\substack{w \in L \\ w+z \neq 0}}(w+z)^{-n}|w+z|^{-s}\right|_{s=0},
$$

where the value at $s=0$ is to be understood in the sense of analytic continuation. These functions are periodic with respect to $L, E_{2 n}$ is even, and $E_{2 n+1}$ is odd. They satisfy

$$
\sum_{k \in L / c L} E_{n}\left(\frac{k}{c}+z\right)=c^{n} E_{n}(c z)
$$

for every $c \in \mathfrak{o}, c \neq 0$. If $\mathfrak{p}(z)$ denotes the Weierstrass $\mathfrak{p}$-function with respect to $L$, then

$$
\mathfrak{p}(z)=E_{2}(z)-E_{2}(0), \quad z \notin L .
$$

Let, for $a, c \in \mathfrak{o}$ with $c \neq 0$,

$$
D(a, c)=\frac{1}{c} \sum_{k \in L / c L} E_{1}\left(\frac{a k}{c}\right) E_{1}\left(\frac{k}{c}\right) .
$$

Define the map $\Phi=\Phi_{L}: S L(2, \mathfrak{o}) \rightarrow C$ by 


$$
\Phi\left(\begin{array}{ll}
a & b \\
c & d
\end{array}\right)= \begin{cases}E_{2}(0) I\left(\frac{a+d}{c}\right)-D(a, c), & c \neq 0 \\
E_{2}(0) I\left(\frac{b}{d}\right), & c=0\end{cases}
$$

where $I(z)=z-\bar{z}$. Then

$$
\Phi(A B)=\Phi(A)+\Phi(B), \quad A, B \in S L(2, \mathfrak{v}),
$$

i.e., $\Phi$ is a homomorphism. Let $g_{2}$ and $g_{3}$ be the coefficients of the equation

$$
\mathfrak{p}^{\prime 2}=4 \mathfrak{p}^{3}-g_{2} \mathfrak{p}-g_{3} .
$$

If both $g_{2}$ and $g_{3}$ belong to the field $F=\boldsymbol{Q}(j)$ of the $j$-invariant $j=$ $12^{3} g_{2}^{3} /\left(g_{2}^{3}-27 g_{3}^{2}\right)$ of $L$, then the values of $\sqrt{D^{-1}} \Phi$ are contained in $F$ (see also [2]). If $g_{2}$ and $g_{3}$ are both integral, then the values of $2 \Phi$ are integral. Assume $D<-4$. Then $E_{2}(0) \neq 0$. Since $\Phi_{\lambda L}=\lambda^{-2} \Phi_{L}$ and

$$
\Phi\left(\begin{array}{cc}
1 & b \\
1
\end{array}\right)=\sqrt{D} E_{2}(0) \quad \text { if } \quad b= \begin{cases}(1+\sqrt{D}) / 2, & D \equiv 1(\bmod 4) \\
\sqrt{D} / 2, & D \equiv 0(\bmod 4) .\end{cases}
$$

the values of $\sqrt{D^{-1}} E_{2}(0)^{-1} \Phi$ depend only on the equivalence class of $L$ and belong to $F$. In general they are not integral, as is seen from the numerical example for the case $D=-23$ in [6].

5. To apply Proposition 1 to $D(a, c)$, we prepare some congruences for division values of $E_{1}$ and $E_{2}$. For the rest of the paper we assume

$$
D \equiv 1 \quad(\bmod 8) \text {. }
$$

Let $\psi$ be a 4 -division point of $C / L$ such that

$$
2 \mathfrak{o}=\{m \in \mathfrak{o} ; 2 m \psi=0\} .
$$

Put

$$
t=\frac{12 \mathfrak{p}(2 \psi)}{\mathfrak{p}(\psi)-\mathfrak{p}(2 \psi)}
$$

and

$$
T(z)=\frac{\mathfrak{p}(\psi)-\mathfrak{p}(2 \psi)}{\mathfrak{p}(z)-\mathfrak{p}(2 \psi)}
$$

Because 2 splits in $K$ the choice of $2 \psi$ is unique and $t$ is determined by 
$L$ up to the sign. We use the following known facts concerning $t$ and $T(z)$ (Fueter [1]).

LEMma 1. Both $t$ and $T(\alpha)$ are algebraic integers prime to 2 if $\alpha \notin L$ and $n \alpha \in L$ with an odd integer $n$.

LEMma 2. (i) If $\alpha \notin L$ and $n \alpha \in L$ with an odd integer $n$, then $\mathfrak{p}(2 \psi)^{-1} \mathfrak{p}(\alpha)$ is algebraic and

$$
\mathfrak{p}(2 \psi)^{-1} \mathfrak{p}(\alpha) \equiv 1 \quad(\bmod 4) .
$$

(ii) $\mathfrak{p}(2 \psi)^{-1} E_{2}(0)$ is algebraic and

$$
\mathfrak{p}(2 \psi)^{-1} E_{2}(0) \equiv-1 \quad(\bmod 4) .
$$

Proof. (i) follows from Lemma 1 and

$$
\frac{\mathfrak{p}(\alpha)}{\mathfrak{p}(2 \psi)}-1=\frac{12}{t T(\alpha)} \text {. }
$$

Let $\mu \in \mathfrak{D}$ with $\mu \equiv \sqrt{D}(\bmod 8)$. We see from (5) and (6) that

$$
\left(\mu^{2}-|\mu|^{2}\right) E_{2}(0)=\sum_{k \in L / \mu L-\{0\}} \mathfrak{p}\left(\frac{k}{\mu}\right)
$$

hence

$$
\frac{1}{2}\left(\mu^{2}-|\mu|^{2}\right) E_{2}(0)=\sum_{\substack{k \in L / \mu L-\{0\} \\ k \bmod \pm 1}} p\left(\frac{k}{\mu}\right) .
$$

Devide both sides by $\mathfrak{p}(2 \psi)$ and use (i). The asserted congruence of (ii) follows from

$$
\begin{array}{ll}
\frac{1}{2}\left(\mu^{2}-|\mu|^{2}\right) \equiv 1 \quad(\bmod 4), \\
\frac{1}{2}\left(|\mu|^{2}-1\right) \equiv-1 \quad(\bmod 4) .
\end{array}
$$

Lemma 3. Let $\alpha$ be a point of $\boldsymbol{C} / L$ of finite, odd order $>1$. Then $E_{2}(0)^{-1 / 2} E_{1}(\alpha)$ is algebraic and

$$
E_{2}(0)^{-1 / 2} E_{1}(\alpha) \equiv 1 \quad(\bmod 2)
$$

Proof. Denote by $n$ the order of $\alpha$. By Lemma 2,

$$
E_{2}(0)^{-1} \mathfrak{h}(k \alpha) \equiv-1 \quad(\bmod 4),
$$


for $1 \leq k \leq n-1$. Note that $a \equiv 1(\bmod 2)$ if and only if $a^{2} \equiv 1(\bmod 4)$. Then the assertions follow from the following identities (cf. [5], [6]):

$$
\begin{aligned}
& n E_{1}(\alpha)=\sum_{k=1}^{n-2}\left(E_{1}(k \alpha)+E_{1}(\alpha)-E_{1}((k+1) \alpha)\right), \\
& \left(E_{1}(k \alpha)+E_{1}(\alpha)-E_{1}((k+1) \alpha)\right)^{2}=\mathfrak{p}(k \alpha)+\mathfrak{p}(\alpha)+\mathfrak{p}((k+1) \alpha) .
\end{aligned}
$$

6. From [6] and [7], we know that there is a homomorphism $\chi$ : $S L(2, \mathfrak{v}) \rightarrow Z / 8 Z$ which is uniquely characterized by

$$
\chi\left(\begin{array}{ll}
1 & 1 \\
& 1
\end{array}\right)=0
$$

and

$$
\chi\left(\begin{array}{ll}
a & b \\
c & d
\end{array}\right)=2 \varepsilon+2\left(1-\left(\frac{2 a}{c}\right)\right)+\operatorname{tr}\left(\frac{(a+d) c}{\sqrt{D}}\right) \quad \bmod 8
$$

for $c \equiv 1(\bmod 2)$. Here,

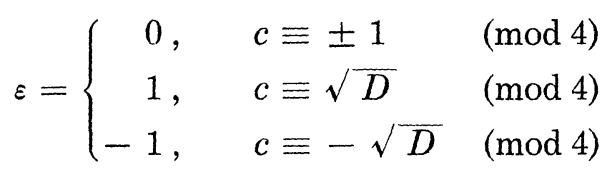

and we agree that $(0 / \pm 1)=1$. This homomorphism $\chi$ describes the eighth roots of unity which occur in the transformation formula of a certain theta series. We note here that $\chi$ depends on the choice of the square root $\sqrt{D}$ of $D$; if we change $\sqrt{D}$ to $-\sqrt{D}$, then $\chi$ changes to $-\chi$.

Theorem 1. For every $A \in S L(2, \mathfrak{v})$,

$$
\sqrt{D}^{-1} E_{2}(0)^{-1} \Phi(A) \equiv \chi(A) \quad(\bmod 8) .
$$

Remark. Although $\chi(A)$ is a class of $Z / 8 Z$ the above congruence obviously makes sense if we consider $\chi(A)$ as a representative in $Z$ of the class.

Proof. It suffices to prove the above under the assumption $A=$ $\left(\begin{array}{ll}a & b \\ c & d\end{array}\right), c \equiv 1(\bmod 2)$. Let $\alpha$ be a primitive $c$-division point of $C / L$. By Lemma 3 we can apply Proposition 1 with $\mathfrak{c}=c \mathfrak{b}$ and

$$
f(k)=E_{2}(0)^{-1 / 2} E_{1}(k \alpha) \text {. }
$$

We get, by (4), 


$$
c E_{2}(0)^{-1} D(a, c) \equiv 2\left(1-\left(\frac{2 a}{c}\right)\right)-1+\left(\frac{-1}{c}\right) \quad(\bmod 8) .
$$

Because 2 splits in $K, c^{2} \equiv 1(\bmod 8)$. Therefore

$$
\sqrt{D}^{-1} I\left(\frac{a+d}{c}\right) \equiv \operatorname{tr}\left(\frac{(a+d) c}{\sqrt{D}}\right) \quad(\bmod 8)
$$

Hence,

$$
\begin{aligned}
& \sqrt{\bar{D}^{-1}} E_{2}(0)^{-1} \Phi(A) \\
& \quad \equiv \operatorname{tr}\left(\frac{(a+d) c}{\sqrt{D}}\right)-2\left(1-\left(\frac{2 a}{c}\right)\right)+\frac{1}{c \sqrt{D}}\left(1-\left(\frac{-1}{c}\right)\right) \quad(\bmod 8) .
\end{aligned}
$$

The value $(-1 / c)$ is 1 if $\varepsilon=0$ and -1 if $\varepsilon \neq 0$. Moreover $c \sqrt{D} \equiv \varepsilon$ $(\bmod 4)$ if $\varepsilon \neq 0$. This completes the proof.

7. By Lemma 1 and Lemma 2, (ii), the number $12 \sqrt{D} E_{2}(0)(\mathfrak{p}(\psi)-$ $\mathfrak{p}(2 \psi))^{-1}$ is algebraic and prime to 2 . Hence we obtain from Theorem 1 the congruence in the next theorem.

Theorem 2. For every $A \in S L(2, \mathfrak{o})$,

$$
\frac{12}{\mathfrak{p}(\psi)-\mathfrak{p}(2 \psi)} \Phi(A) \equiv \frac{12 \sqrt{D} E_{2}(0)}{\mathfrak{p}(\psi)-\mathfrak{p}(2 \psi)} \chi(A) \quad(\bmod 8)
$$

The left hand side and the coefficient of $\chi$ are of the form $\sqrt{-1} \times$ (an integer of $F$ ).

Proof. Because of (7) it suffices to prove the second assertion for the left hand side of (8). First we see the integrality. Put

$$
\gamma=\mathfrak{p}(\psi)-\mathfrak{p}(2 \psi), \quad \delta=\mathfrak{p}(2 \psi)
$$

and

$$
T_{1}(z)=\gamma^{-1 / 2} \frac{d}{d z} T(z)
$$

Then,

$$
T_{1}^{2}=T\left(4 T^{2}+t T+4\right),
$$

cf. [1]. From this follows that

$$
\mathfrak{p}^{2}=4 \mathfrak{p}^{3}-g_{2} \mathfrak{p}-g_{3}
$$

with 


$$
\begin{aligned}
& g_{2}=12 \delta^{2}-4 \gamma^{2}=12^{-1} \gamma^{2} t^{2}-4 \gamma^{2}, \\
& g_{3}=4 \gamma^{2} \delta-8 \delta^{3}=3^{-1} \gamma^{3} t-6^{-3} \gamma^{3} t^{3} .
\end{aligned}
$$

Recall that the numbers we are interested in do not change when we replace the pair $(L, \psi)$ by $(\lambda L, \lambda \psi)$. Taking $\lambda=\sqrt{6 \gamma^{-1}}$, we may assume $\gamma=6$. Then

$$
g_{2}=3 t^{2}-144, \quad g_{3}=\left(72-t^{2}\right) t
$$

and the left hand side of (8) becomes $2 \Phi(A)$. Since $g_{2}$ and $g_{3}$ are integral, it is also integral. To prove that it is of the form $\sqrt{-1} \mu(\mu \in F)$, it suffices to show that $g_{2},|D|^{1 / 2} g_{3} \in F$ for the values of $g_{2}$ and $g_{3}$ given in (10). This condition is equivalent to $|D|^{1 / 2} t \in F$. We may assume $L=\bar{L}$. It is known (cf. [1]) that $t^{2}$ belongs to the Hilbert class field $F(\sqrt{D})$ of $K$ and that $t$ generates over $K$ the ray class field modulo 4 of $K$, which is $F(\sqrt{D}, \sqrt{-1})$ in our case. It follows that $t \in F\left(|D|^{1 / 2}\right), t^{2} \in F$ and $t \notin F$. Hence $|D|^{1 / 2} t \in F$. This concludes the proof.

If our lattice $L$ satisfies

$$
(\mathfrak{p}(\psi)-\mathfrak{p}(2 \psi))^{2}=144|D|,
$$

then

$$
\frac{1}{\sqrt{D}} \Phi(A) \equiv E_{2}(0) \chi(A) \quad(\bmod 8)
$$

and $\sqrt{D^{-1}} \Phi(A)$ and $E_{2}(0)$ are integers of $F$. Furthermore, $E_{2}(0)$ is prime to 2. It can be seen that the lattices considered in [6], §5 satisfy (11). Hence we have proved Conjecture 1 of Sczech [6]. The condition (11) is independent of the choice of $\psi$.

\section{REFERENCES}

[1] R. Fueter, Vorlesungen über die singulären Moduln und die komplexe Multiplikation der elliptischen Funktionen I, II, Teubner, Leipzig-Berlin, 1924, 1927.

[2] H. Ito, On a property of elliptic Dedekind sums, J. Number Theory, 27 (1987), $17-21$.

[ 3 ] H. Rademacher and E. Grosswald, Dedekind sums, Carus Mathematical Monographs, No. 16, Mathematical Assoc. America, Washington D. C., 1972.

[4] H. Reichardt, Eine Bemerkung zur Theorie des Jacobischen Symbols, Math. Nachr., 19 (1958), 171-175.

[ 5 ] R. Sczech, Dedekindsummen mit elliptischen Funktionen, Invent. Math., 76 (1984), $523-551$. 
[6] —- Dedekind sums and power residue symbols, Compositio Math., 59 (1986), $89-112$.

[ 7 ] — , Theta functions on the hyperbolic three space, Kokyuroku RIMS, Kyoto Univ., No. 603 (1987), pp. 9-20.

The Institute for Advanced Study

Princeton, NJ 08540

USA

and

Nagoya University

Chikusa-ku, Nagoya, 464

Japan

Current address:

Department of Mathematics

College of Arts and Sciences

University of Tokyo

Tokyo 153, Japan 